University of Nebraska - Lincoln

DigitalCommons@University of Nebraska - Lincoln

Faculty Publications, Department of Physics and Astronomy

Research Papers in Physics and Astronomy

$7-1-2004$

\title{
Focused Ion Beam Milled CoPt Magnetic Force Microscopy Tips for High Resolution Domain Images
}

L. Gao

University of Nebraska-Lincoln, Igao@unlserve.unl.edu

Lanping Yue

University of Nebraska-Lincoln, lyue2@unl.edu

T. Yokota

University of Nebraska-Lincoln, TYOKOTA2@unl.edu

Ralph Skomski

University of Nebraska-Lincoln, rskomski2@unl.edu

Sy-Hwang Liou

University of Nebraska-Lincoln, sliou@unl.edu

See next page for additional authors

Follow this and additional works at: https://digitalcommons.unl.edu/physicsfacpub

Part of the Physics Commons

Gao, L.; Yue, Lanping; Yokota, T.; Skomski, Ralph; Liou, Sy-Hwang; Takahoshi, H.; Saito, Hitoshi; and Ishio, S., "Focused Ion Beam Milled CoPt Magnetic Force Microscopy Tips for High Resolution Domain Images" (2004). Faculty Publications, Department of Physics and Astronomy. 34.

https://digitalcommons.unl.edu/physicsfacpub/34

This Article is brought to you for free and open access by the Research Papers in Physics and Astronomy at DigitalCommons@University of Nebraska - Lincoln. It has been accepted for inclusion in Faculty Publications, Department of Physics and Astronomy by an authorized administrator of DigitalCommons@University of Nebraska Lincoln. 


\section{Authors}

L. Gao, Lanping Yue, T. Yokota, Ralph Skomski, Sy-Hwang Liou, H. Takahoshi, Hitoshi Saito, and S. Ishio 


\title{
Focused Ion Beam Milled CoPt Magnetic Force Microscopy Tips for High Resolution Domain Images
}

\author{
L. Gao, L. P. Yue, T. Yokota, R. Skomski, S. H. Liou, H. Takahoshi, H. Saito, and S. Ishio
}

\begin{abstract}
High-coercivity CoPt magnetic force microscope tips have been modified by focused ion beam milling to improve the resolution of magnetic domain images. The magnetic materials around the apex have been removed, leaving a 30-nm diameter magnetic particle at the tip end. Due to the smaller amount of magnetic material, the stray field from this new tip is significantly reduced, and the spatial resolution of the magnetic domain images is improved. The tip is used to obtain high-resolution domain images of a $\mathrm{CoCrPt}-\mathrm{SiO}_{2} / \mathrm{Ru}$ perpendicular recording medium with linear recording densities from 800 to $1000 \mathrm{kfci}$. Magnetic patterns of $900 \mathrm{kfci}$, corresponding to a bit size of $28 \mathrm{~nm}$, are well resolved. From the analysis of the power spectrum of the track profiles for these images, a spatial resolution as good as $\mathbf{1 1}$ nm under ambient conditions with a commercial magnetic force microscope is achieved.
\end{abstract}

Index Terms-Domain images, focused ion beam technology, magnetic force microscopy, magnetic recording.

\section{INTRODUCTION}

$\mathbf{M}$ AGNETIC force microscopy (MFM) is a powerful technique to study the magnetic domain structures for high density magnetic recording media [1]. With the now widely available commercial microscopes and magnetic tips, images with 50-nm resolution are routinely obtained. However, there remains the challenge of obtaining images with higher resolution, which are of interest, for example, in high-density magnetic recording [2].

The tip is one of the most critical parts of the MFM. The requirements for high-resolution MFM tips are a well-defined magnetization direction, small stray fields, and sharp ends. A well-defined magnetization direction is achieved by using tips with highly coercive magnetic particles, which also assures that the magnetization direction of the tip remains undisturbed by high stray field samples. This is useful for domain imaging under an applied magnetic field. It has been shown by electron holography that the magnetic flux originates not only from the tip end but also from other magnetic parts of the tip [3]. The magnetic dipole interaction is a long-range interaction that

Manuscript received October 15, 2003. This work was supported by the National Science Foundations under MRSEC Awards DMR-0213808 and DMR0116780 and by the Army Research Office under Grant DAAD 19-03-1-0298. T. Yokota is supported by a Grant-in-Aid for JSPS Fellows, no. 00000652.

L. Gao, L. P. Yue, T. Yokota, R. Skomski, and S. H. Liou are with the Department of Physics and Astronomy and Center for Materials Research and Analysis, University of Nebraska, Lincoln, NE 68588 USA (e-mail: lgao@unlserve.unl.edu; 1yue2@unl.edu; TYOKOTA2@unl.edu; rskomski@unlserve.unl.edu; sliou@unl.edu).

H. Takahoshi, H. Saito, and S. Ishio are with the Department of Materials Science and Engineering, Akita University, Akita 010, Japan (email: takahosi@ipc.akita-u.ac.jp; hsaito@jpc.akita-u.ac.jp; ishio@jpc.akita-u.ac.jp).

Digital Object Identifier 10.1109/TMAG.2004.829173 includes not only the contribution between the sample and the magnetic material near the tip but also that from extended areas of the tip. Small stray fields can be achieved by reducing the magnetic volumes of the tip interacting with a magnetic sample. Small volumes and sharp ends are also useful to provide a better spatial resolution of the MFM images.

One way to enhance the resolution of MFM images is to improve the magnetic tips by removing magnetic materials around the tip end. Focused ion beam (FIB) milling has been used previously to realize this MFM-tip modification. Folks et al. [4] made a hole as small as $20 \mathrm{~nm}$ at the apex of a CoCr tip to improve the resolution of in-plane components of the stray field and to identify a 50-nm transition. Liu et al. [5] sharpened the tip using an FIB and then coated a 30-nm exchanged coupled antiferromagnet-ferromagnet multilayer on the tip. Square patterns on a perpendicular media with a period of $60 \mathrm{~nm}$ could be recognized. Phillips et al. [6] formed a planar magnetic element with high shape anisotropy and an end radius of less than $25 \mathrm{~nm}$. A spatial resolution of $30 \mathrm{~nm}$ was reported. In this work, we use a focused ion beam to mill a permanent magnet CoPt tip, reducing the tip end diameter to $30 \mathrm{~nm}$ and presenting a lateral resolution of $11 \mathrm{~nm}$. As far as we know, it is the best resolution reported for commercial MFM in ambient condition.

\section{EXPERIMENT}

The MFM tip was fabricated using a CoPt permanent magnetic film coating and FIB milling. The $30-\mathrm{nm}$-thick CoPt film is deposited on commercially available batch fabricated, micromachined cantilevers and then annealed at $650 \circ \mathrm{C}$ for $10 \mathrm{~h}$ to form tetragonal $\left(\mathrm{L}_{0}\right)$ phase. Tetragonal $\left(\mathrm{L1}_{0}\right) \mathrm{CoPt}$ is an excellent material due to the alloy's chemical stability and its high magnetic anisotropy of about $4 \times 10^{7} \mathrm{ergs} / \mathrm{cm}^{3}$. Its saturation magnetization is about $800 \mathrm{emu} / \mathrm{cm}^{3}$ [7], and the material's anisotropy has allowed us to produce CoPt tips having coercivities as high as $15 \mathrm{kOe}$.

Fig. 1 shows that there are many CoPt magnetic particles visible on the tip. The CoPt coated tip is then modified by FIB milling with $\mathrm{Ga}^{+}$ions possessing an energy of $30 \mathrm{keV}$ with a 4-pA beam current. It is known that the $\mathrm{Ga}^{+}$ions degrade the magnetic properties of the magnetic material [8]. To reduce its exposure to the ions and to avoid damage, we mill the tip from the side, that is, the ion beam direction is perpendicular to the tip axis. The majority of the CoPt film is milled away, leaving a 30-nm diameter magnetic particle on the very end of the tip (Fig. 1). The distance from the tip-end to the large amount of magnetic particles on the tip is around $600 \mathrm{~nm}$. Their contributions to the tip-sample interaction are negligible due to the magnetic interaction dropping rapidly with distance. 


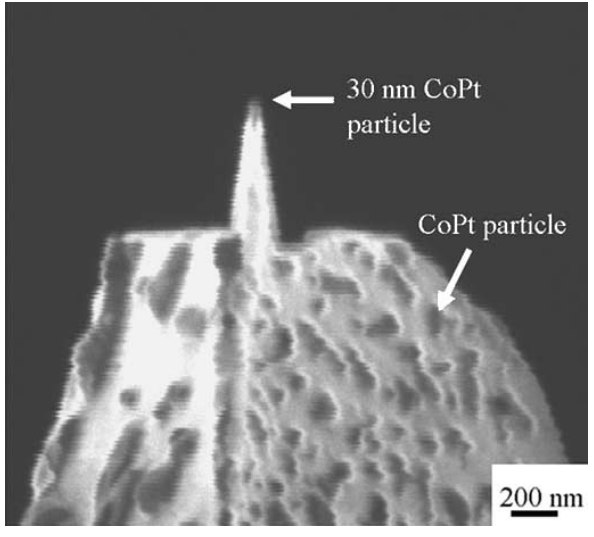

Fig. 1. FIB milled MFM tip shows a magnetic particle with a diameter of 30 $\mathrm{nm}$ at the tip end.

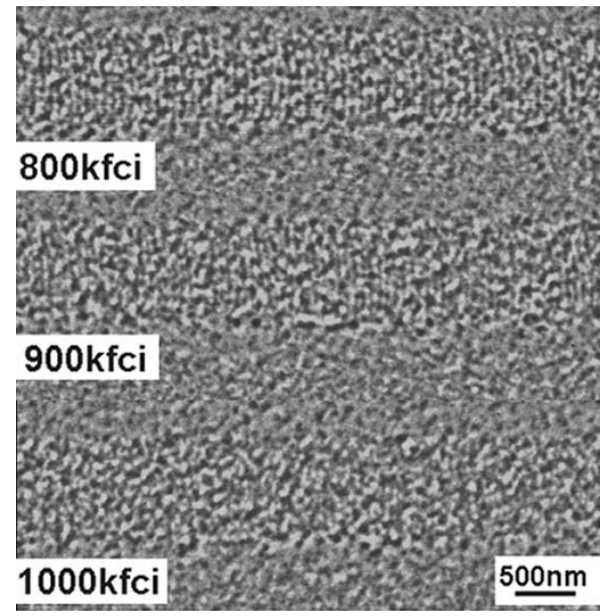

Fig. 2. Magnetic domain images of recording tracks with linear densities from 800 to $1000 \mathrm{kfci}$.

\section{RESULTS}

To characterize the resolution of the FIB tip, we used a high-density $\mathrm{CoCrPt}: \mathrm{SiO}_{2} / \mathrm{Ru}$ perpendicular magnetic recording medium. The medium was chosen as a test sample because it consists of periodic fine domain structures. MFM images were obtained at room temperature, in air, using a commercial MFM operated in tapping/lift mode at a lift height of $5 \mathrm{~nm}$. Fig. 2 shows the domain images of recording tracks with linear recording densities from 800 to $1000 \mathrm{kfci}$. Tracks of 800,900 , and $1000 \mathrm{kfci}$ correspond to bit sizes of 32, 28, and $25 \mathrm{~nm}$, respectively. The MFM image of the 800-kfci track presents well-resolved recording bits. Track densities up to 900 kfci are clearly visible by MFM. The visibility of the $1000 \mathrm{kfci}$ track transitions is much less pronounced.

Magnetic coupling across grains forms magnetic interaction clusters. From the above MFM images, the bit size for 800and 900-kfci tracks is smaller than the magnetic cluster size for regions outside recording track and can be well resolved. This indicates that the magnetic coupling in these magnetic grains is weak.

The resolution of the MFM can be characterized in real space by its "point response" [9] or in Fourier transform space by its wave-vector (spatial frequency) response [1], [10]. Essentially,
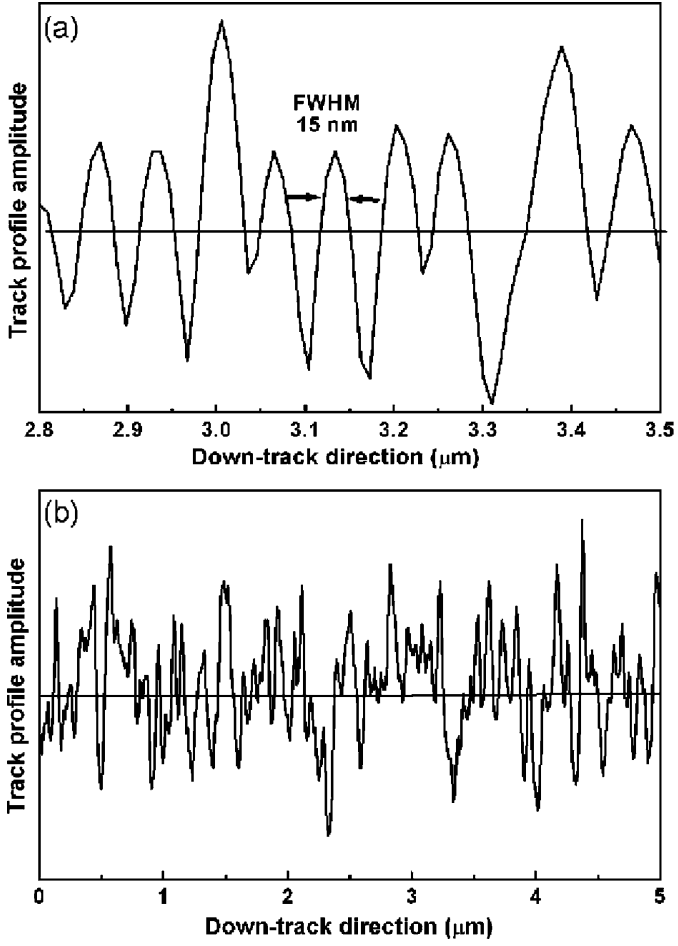

Fig. 3. (a) Averaged profile and (b) single profile of a 900-kfci track. The FWHM of the peak is $15 \mathrm{~nm}$, corresponding to the real space resolution.

the two approaches are equivalent. Fig. 3(a) shows the averaged track profile for $900 \mathrm{kfci}$. A full width at half maximum (FWHM) as small as $15 \mathrm{~nm}$ is demonstrated, corresponding to the real space resolution. In this work, we use also a Fourier transform method by defining a high spatial frequency cutoff, corresponding to the minimum detectable wavelength $\lambda_{c}$, as the MFM resolution [1].

The response of the MFM is a function of spatial frequency components in the stray field. Such a response curve is called the tip transfer function (TTF) [1]. As shown in [1], long, thin, slablike shapes are characterized by the transfer functions

$$
\begin{aligned}
F(k, z)= & -\frac{\propto_{0} M_{\text {Sam }} M_{\text {Tip }}}{k} e^{-k z}\left(1-e^{-k t}\right) \\
& \times \sin \left(\frac{k b}{2}\right)\left(1-e^{-k l}\right) \\
F^{\prime}(k, z)= & -\propto_{0} M_{\text {Sam }} M_{\text {Tip }} e^{-k z}\left(1-e^{-k t}\right) \\
& \times \sin \left(\frac{k b}{2}\right)\left(1-e^{-k l}\right) .
\end{aligned}
$$

Here, $M_{\text {Sam }}$ and $M_{\text {Tip }}$ are the moments of the sample and the tip, respectively, $k$ is the spatial frequency, $z$ is the tip-sample distance; $t$ is the thickness of the sample film, and $b$ and $l$ are the magnetic coating thickness and length of the tip.

The MFM response is proportional to the magnetization of tip and sample. It has an exponential decay $e^{-k z}$ with increasing tip-sample separation. This explains the importance of the sample-tip distance in the high-resolution MFM images. The factor $\left(1-e^{-k t}\right)$ describes the thickness loss, resulting from the superposition of the stray fields of the magnetic charge sheets on the top and the bottom side of the measured layer [1]. The term $\left(1-e^{-k l}\right)$ describes the low-frequency 


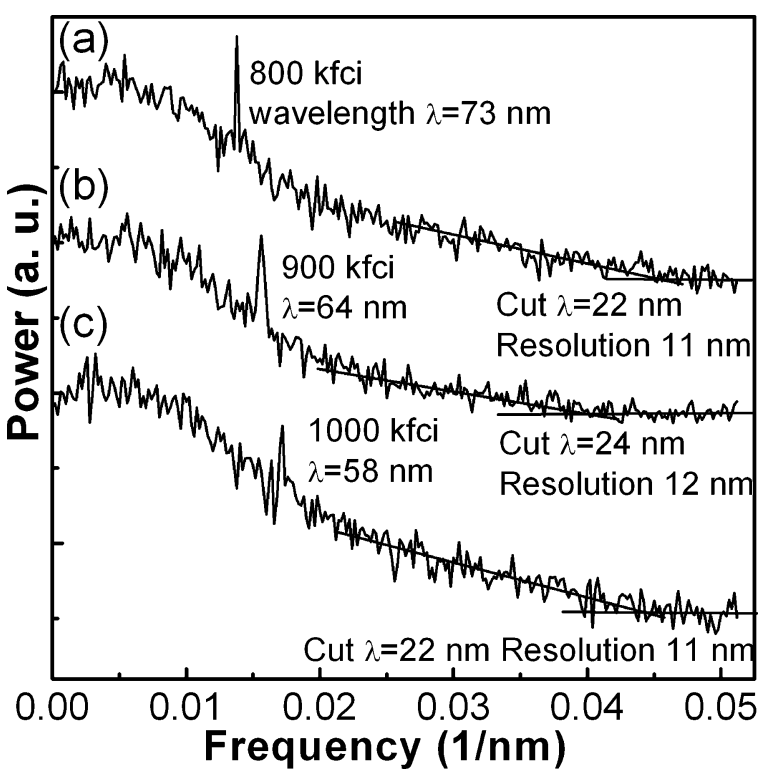

Fig. 4. MFM analysis method is used to analyze the power spectrum of the profile by Fourier transformation. The resolution is (a) $11 \mathrm{~nm}$ for the $800-\mathrm{kfci}$ track, (b) $12 \mathrm{~nm}$ for $900 \mathrm{kfci}$, and (c) $11 \mathrm{~nm}$ for $1000 \mathrm{kfci}$

behavior of the MFM. The $\sin (k b / 2)$ term establishes another limit for high-resolution detection and means that the transfer function is zero at $\mathrm{n} \lambda=\mathrm{b}$. The MFM response drops below the noise level below this wavelength, which is the minimum detectable wavelength by the MFM. To lower the wavelength, the magnetic coating thickness should be reduced as much as possible.

The Fourier transformation is done to get the power spectrum of the profile [11]. Fig. 3(b) shows an example of a single profile for domain images of $900 \mathrm{kfci}$ that was used in obtaining power spectra. The analyzed area is selected from the recorded bit area parallel to the track direction. We have averaged ten power spectra after performing a Fourier transform on a single profile to obtain the resolution. Fig. 4(a) shows the power spectrum of the 800-kfci track. The peak corresponds to the recorded signal with a wave length of $73 \mathrm{~nm}$ (which is double the recording bit size). This value is higher than the calculated value of $64 \mathrm{~nm}$ for $800 \mathrm{kfci}$. This may be due to inaccuracies in the recording process. As shown in Fig. 4(c), the peak corresponds to the recorded signal of a 1000-kfci track with a wavelength of $58 \mathrm{~nm}$ and is only resolved by Fourier transform. In Fig. 4(a), a wavelength cutoff of $22 \mathrm{~nm}$ is obtained from the intersection of the signal and the noise. This corresponds to an MFM image resolution of $11 \mathrm{~nm}$, which is half the wavelength. For the 900- and 1000-kfci tracks, the resolutions obtained from the wavelength cutoffs are 12 and $11 \mathrm{~nm}$, respectively, as shown in Fig. 4(b) and (4c). The error of the resolution is about $2 \mathrm{~nm}$, which is due to the inaccuracies of drawing the signal line and the noise line.

\section{CONCLUSIONS}

In this work, we used an FIB to mill away the CoPt magnetic film covering an MFM tip, leaving a 30-nm diameter particle at the tip end. This reduces the magnetic moment interacting with the sample and improves the resolution of the MFM images. Domain images of magnetic recording media with densities of up to $900 \mathrm{kfci}$ are well resolved using these FIB-milled MFM tips. From analysis of the averaged track profile for $900 \mathrm{kfci}$, a real space resolution as small as $15 \mathrm{~nm}$ is demonstrated. From the analysis of the power spectrum of the track profiles for these images, we show a spatial resolution as good as $11 \mathrm{~nm}$ under ambient conditions with a commercial magnetic force microscope.

\section{REFERENCES}

[1] S. Porthun, L. Abelmann, and J. C. Lodder, "Magnetic force microscopy of thin film media for high density magnetic recording," J. Magn. Magn. Mater, vol. 182, pp. 238-273, 1998.

[2] K. Stoev, F. Liu, Y. Chen, X. Dang, P. Luo, J. Chen, J. Wang, K. Kung, M. Lederman, M. Re, G. Choe, J. N. Zhou, and M. Yu, "Demonstration and characterization of $130 \mathrm{~Gb} / \mathrm{in}^{2}$ magnetic recording systems," J. Appl. Phys., vol. 93, pp. 6552-6554, May 2003.

[3] S. Signoretti, C. Beeli, and S. H. Liou, "Electron holography quantitative measurements on magnetic force microscopy probes," J. Magn. Magn. Mater, submitted for publication.

[4] L. Folks, M. E. Best, P. M. Rice, B. D. Terris, D. Weller, and J. N. Chapman, "Perforated tips for high-resolution in-plane magnetic force microscopy," Appl. Phys. Lett., vol. 76, pp. 909-911, Feb. 2000.

[5] Z. Liu, Y. Dan, Q. Jinjun, and Y. Wu, "Magnetic force microscopy using focused ion beam sharpened tip with deposited antiferro-ferromagnetic multiple layers," J. Appl. Phys., vol. 91, pp. 8843-8845, May 2002.

[6] G. N. Phillips, M. Siekman, L. Abelmann, and J. C. Lodder, "High resolution magnetic force microscopy using focused ion beam modified tips," Appl. Phys. Lett., vol. 81, pp. 865-867, July 2002.

[7] S. H. Liou, S. Huang, E. Klimek, R. D. Kirby, and Y. D. Yao, "Enhancement of coercivity in nanometer-size CoPt crystallites," J. Appl. Phys., vol. 85, pp. 4334-4336, Apr. 1999

[8] C. T. Rettner, S. Anders, T. Thomson, M. Albrecht, Y. Ikeda, M. E. Best, and B. D. Terris, "Magnetic characterization and recording properties of patterned $\mathrm{Co}_{70} \mathrm{Cr}_{18} \mathrm{Pt}_{12}$ perpendicular media," IEEE Trans. Magn., vol. 38, pp. 1725-1730, July 2002.

[9] D. Rugar, H. J. Mamin, P. Guethner, S. E. Lambert, J. E. Stern, I. McFadyen, and T. Yogi, "Magnetic force microscopy: General principles and application to longitudinal recording media," J. Appl. Phys., vol. 68, pp. 1169-1183, Aug. 1990.

[10] H. J. Jug, B. Stiefel, P. J. A. van Schendel, A. Moser, R. Hofer, S. Martin, J. J. Guntherodt, S. Porthum, L. Abelmann, J. C. Lodder, G. Bochi, and R. C. Ohandley, "Quantitative magnetic force microscopy on perpendicularly magnetized samples," J. Appl. Phys., vol. 83, pp. 5609-5620, June 1998.

[11] J. Chen, H. Saito, S. Ishio, and K. Kobayashi, "Analysis of two-dimensional medium noise and magnetic cluster with MFM for $\mathrm{Co}_{82} \mathrm{Cr}_{13} \mathrm{Ta}_{5}$ longitudinal magnetic recording media," J. Magn. Magn. Mater., vol. 188, pp. 260-267, 1998. 